\title{
Utility of Activated Nitriles in the Synthesis of Novel Heterocyclic Compounds with Antitumor Activity
}

\author{
Asmaa S. Salman \\ Department of Chemistry, Faculty of Science, Al-Azhar University, Girls' Branch, Nasr City, Cairo, Egypt \\ Correspondence should be addressed to Asmaa S. Salman; salman_2007_ok@yahoo.com
}

Received 23 July 2013; Accepted 19 August 2013

Academic Editor: Joseph E. Saavedra

Copyright ( 2013 Asmaa S. Salman. This is an open access article distributed under the Creative Commons Attribution License, which permits unrestricted use, distribution, and reproduction in any medium, provided the original work is properly cited.

\begin{abstract}
Reaction of cyanoacetic acid hydrazide (1) with 4-methoxyacetophenone and 4-chlorobenzaldehyde (2a,b) afforded the corresponding 2-cyanoacetohydrazide derivatives $(\mathbf{3} \mathbf{a}, \mathbf{b})$ respectively. The latter compounds were utilized as a key intermediate for the synthesis of new heterocyclic compounds. Newly synthesized compounds were characterized by elemental analyses and spectral data. The antitumor evaluation of some newly synthesized compounds was screened in vitro against human breast cancer cell line (MCF-7).
\end{abstract}

\section{Introduction}

In many reports, hydrazide-hydrazones are considered to be good candidates for different pharmaceutical applications, where such compounds were considered to exert anticonvulsant [1], analgesic [2], anti-inflammatory [3], antiplatelet [4], antimalarial [5], antimicrobial [6], antitumoral [7, 8], vasodilator [9], and antiviral activity [10]. With the aim of obtaining new hydrazide-hydrazones with such wide spectrum of pharmaceutical applications, in this research, synthesis of a series of hydrazide-hydrazones and some of their heterocyclic transformations, followed by antitumor evaluations of newly synthesized products was done.

\section{Experimental}

All melting points were determined in open glass capillaries on a Gallenkamp apparatus and are uncorrected. IR spectra $\left(\mathrm{cm}^{-1}\right)$ were recorded on a Pye-Unicam spectrophotometer type 1200 using $\mathrm{KBr}$ discs. ${ }^{1} \mathrm{H}-\mathrm{NMR}$ spectra were recorded on a Varian EM-390 $(90 \mathrm{MHz})$ spectrometer using TMS as an internal standard and DMSO-d6 as a solvent. Chemical shifts were expressed in $\delta$ (ppm) values and mass spectra were determined on Finnigan Incos 500 (70 ev). Elemental analyses were determined using a Parkin-Elmer 240C Microanalyzer. The microanalyses were performed at the Microanalytical Unit, Faculty of Science, Cairo University.
2.1. General Procedure for Synthesis of $\mathbf{3 a}, \boldsymbol{b}$. To a solution of 2cyanoacetohydrazide $(0.99 \mathrm{~g}, 0.01 \mathrm{~mol})$ in ethanol $(20 \mathrm{~mL})$, 4-methoxyacetophenone and/or p-chlorobenzaldehyde $(0.01 \mathrm{~mol})$ was added. The reaction mixture was heated under reflux for $2 \mathrm{~h}$, then left to cool, and poured into ice/ water. The obtained product was filtered, washed with water, and recrystallized from the appropriate solvent to give $\mathbf{3 a}, \mathbf{b}$.

2.1.1. Cyano- $N^{\prime}-[1-(4-m e t h o x y p h e n y l) e t h y l i d e n e] a c e t o h y d r a z-$ ide (3a). Yield, $75 \%$; m.p. $186-188^{\circ} \mathrm{C}$ (ethanol); IR ( $\mathrm{KBr}$, $\mathrm{cm}^{-1}$ ): $3204(\mathrm{NH}), 1675$ (CO), $2256(\mathrm{CN}) ;{ }^{1} \mathrm{H}$ NMR (DMSO: $\delta \mathrm{ppm}$ ): 2.23 (s, $\left.3 \mathrm{H}, \mathrm{CH}_{3}\right), 3.81\left(\mathrm{~s}, 3 \mathrm{H}, \mathrm{OCH}_{3}\right), 4.18(\mathrm{~s}, 2 \mathrm{H}$, $\left.\mathrm{CH}_{2}\right), 10.90$ (s, 1H, NH), 6.93-7.76 (m, 4H Ar-H); MS m/z (\%): $231\left(\mathrm{M}^{+}, 98.11\right), 216$ (27.99), 191 (40.26), 163 (48.64), 148 (80.06), 134 (87.96), 121 (39.49), 119 (56.71), 92 (100). Anal. for $\mathrm{C}_{12} \mathrm{H}_{13} \mathrm{~N}_{3} \mathrm{O}_{2}$ (231.25); Calcd.: C, 62.33; $\mathrm{H}, 5.67 ; \mathrm{N}, 18.17$. Found: C, 62.00; H, 5.33; N, 18.02\%.

2.1.2. $N^{\prime}$-[(4-Chlorophenyl)methylene $]$-2-cyanoacetohydrazide (3b). Yield, $80 \%$; m.p. $210-214^{\circ} \mathrm{C}$ (ethanol); IR $(\mathrm{KBr}$, $\mathrm{cm}^{-1}$ ): $3185(\mathrm{NH}), 1673(\mathrm{CO}), 2258(\mathrm{CN}) ;{ }^{1} \mathrm{H}$ NMR (DMSO, $\delta \mathrm{ppm}$ ): 4.21 (s, $\left.2 \mathrm{H}, \mathrm{CH}_{2}\right), 11.82(\mathrm{~s}, 1 \mathrm{H}, \mathrm{NH}), 7.48-7.99(\mathrm{~m}, 4 \mathrm{H}$ $\mathrm{Ar}-\mathrm{H}), 8.16$ (s, $1 \mathrm{H},=\mathrm{CH}) ; \mathrm{MS} m / z(\%): 221\left(\mathrm{M}^{+}, 2.35\right), 153$ (5.54), 138 (100), 126 (15.89), 76 (15.35). Anal. for $\mathrm{C}_{10} \mathrm{H}_{8} \mathrm{Cl}$ $\mathrm{N}_{3} \mathrm{O}$ (221.64); Calcd.: C, 54.19; H, 3.64; Cl, 16.00; N, 18.96 . Found: C, 54.00; H, 3.42; Cl, 15.82; N, 18.65\%. 
2.2. General Procedure for Synthesis of $\mathbf{4 a}, \boldsymbol{b}$. To a mixture of $3 \mathbf{a}, \mathbf{b}(0.01 \mathrm{~mol})$, and salicylaldehyde $(1.22 \mathrm{~g}, 0.01 \mathrm{~mol})$ in ethanol $(15 \mathrm{~mL})$ were added few drops of TEA. The reaction was heated on water bath for $4 \mathrm{~h}$ and poured into ice water. The obtained product was filtered, washed with water, and recrystallized from the appropriate solvent to give $\mathbf{4 a}, \mathbf{b}$.

2.2.1. 2-Imino- $N^{\prime}$-[1-(4-methoxyphenyl)ethylidene]-2H-chromene-3-carbohydrazide (4a). Yield, 80\%; m.p. $210-212^{\circ} \mathrm{C}$ (ethanol); IR (KBr, cm $\left.{ }^{-1}\right)$ : 3430, $3300(2 \mathrm{NH}), 1671(\mathrm{CO}) ;{ }^{1} \mathrm{H}$ NMR (DMSO, $\delta$ ppm): $3.80\left(\mathrm{~s}, 3 \mathrm{H}, \mathrm{OCH}_{3}\right), 2.48\left(\mathrm{~s}, 3 \mathrm{H}, \mathrm{CH}_{3}\right)$, 6.98-7.83 (m, 9H, Ar-H and $\mathrm{C} 4-\mathrm{H}$ coumarin), 9.27 (s, $1 \mathrm{H}$, $\mathrm{NH}), 13.47$ (s, 1H, NH); MS m/z (\%): $335\left(\mathrm{M}^{+}, 100\right), 320$ (18.43), 148 (3.90), 145 (95.72), 134 (12.36). Anal. for $\mathrm{C}_{19} \mathrm{H}_{17}$ $\mathrm{N}_{3} \mathrm{O}_{3}$ (335.35); Calcd.: C, 68.05; H, 5.11; N, 12.53. Found: C, $68.00 ; \mathrm{H}, 5.09 ; \mathrm{N}, 12.23 \%$.

2.2.2. $\quad N^{\prime}$-[(4-Chlorophenyl)methylene]-2-imino-2H-chromene-3-carbohydrazide(4b). Yield, 75\%; m.p. $170-172^{\circ} \mathrm{C}$ (ethanol); IR (KBr, cm $\left.{ }^{-1}\right): 3422,3310(2 \mathrm{NH}), 1618(\mathrm{CO}) ;{ }^{1} \mathrm{H}$ NMR (DMSO, $\delta$ ppm): 6.94-7.70 (m, 10H, Ar-H, C4-H coumarin and $=\mathrm{CH}), 9.00(\mathrm{~s}, 1 \mathrm{H}, \mathrm{NH}), 11.10(\mathrm{~s}, 1 \mathrm{H}, \mathrm{NH}) ; \mathrm{MS} \mathrm{m} / z(\%)$ : $324\left(\mathrm{M}^{+}-1,1.54\right), 310$ (3.73), 239 (32.03), 165 (100). Anal. for $\mathrm{C}_{17} \mathrm{H}_{12} \mathrm{Cl} \mathrm{N}_{3} \mathrm{O}_{2}$ (325.74); Calcd.: C, 62.68; H, 3.71; Cl, 10.88; N, 12.90. Found: C, 62.38; H, 3.41; Cl, 10.52; N, 12.69\%.

2.3. General Procedure for Synthesis of Arylidene Derivatives $(\mathbf{5 a}-\boldsymbol{d})$. A solution of $\mathbf{3 a}, \mathbf{b}(0.01 \mathrm{~mol})$ in ethanol $(30 \mathrm{~mL})$ was treated with $0.01 \mathrm{~mol}$ of aromatic aldehydes such as 4-methoxybenzaldehyde and/or 4-chlorobenzaldehyde and few drops of TEA. The reaction mixture was heated under reflux for $6 \mathrm{~h}$. The reaction mixture was left to cool at room temperature and poured into ice/water containing few drops of hydrochloric acid, and the formed solid was collected by filtration and recrystallized from the appropriate solvent to give $\mathbf{5 a}-\mathbf{d}$.

2.3.12-Cyano-3-(4-methoxyphenyl)-N'-[1-(4-methoxyphenyl)ethylidene acrylohydrazide(5a). Yield, 60\%; m.p. 158-160 ${ }^{\circ} \mathrm{C}$ (ethanol); IR (KBr, cm ${ }^{-1}$ ): $3367(\mathrm{NH}), 2199(\mathrm{CN}), 1686(\mathrm{CO})$; ${ }^{1} \mathrm{H}$ NMR (DMSO, $\left.\delta \mathrm{ppm}\right): 3.77\left(\mathrm{~s}, 6 \mathrm{H}, 2 \mathrm{OCH}_{3}\right), 2.49(\mathrm{~s}, 3 \mathrm{H}$, $\left.\mathrm{CH}_{3}\right), 6.91-8.17$ (m, 9H, Ar- $\mathrm{H}$ and $=\mathrm{CH}$ proton), 10.10 (s, $1 \mathrm{H}$, $\mathrm{NH}$ ); Anal. for $\mathrm{C}_{20} \mathrm{H}_{19} \mathrm{~N}_{3} \mathrm{O}_{3}$ (349.38; Calcd.: C, 68.75; H, 5.48; N, 12.03. Found: C, 68.55; H, 5.28; N, 12.00\%.

2.3.2. $N^{\prime}$-[(4-Chlorophenyl)methylene]-2-cyano-3-(4-methoxyphenyl)acrylohydrazide (5b). Yield, 65\%; m.p. $168-170^{\circ} \mathrm{C}$ (ethanol); IR (KBr, cm ${ }^{-1}$ ): 3287 (NH), 2209 (CN), 1680 (CO); ${ }^{1} \mathrm{H}$ NMR (DMSO, $\delta$ ppm): 3.87 (s, 3H, $\left.\mathrm{OCH}_{3}\right), 6.91-8.17(\mathrm{~m}$, $10 \mathrm{H}, \mathrm{Ar}-\mathrm{H}$ and $=\mathrm{CH}$ proton), $8.71(\mathrm{~s}, 1 \mathrm{H}, \mathrm{NH}) ; \mathrm{MS} m / z(\%)$ : $339\left(\mathrm{M}^{+}\right.$, 3.15), 201 (100), 186 (93.94), 158 (38.79). Anal. for $\mathrm{C}_{18} \mathrm{H}_{14} \mathrm{Cl} \mathrm{N}_{3} \mathrm{O}_{2}$ (339.77); Calcd.: C, 63.63; H, 4.15; Cl, 10.43; N, 12.37. Found: C, 63.43; H, 4.00; Cl, 10.23; N, $12.00 \%$.

2.3.3. 3-(4-Chlorophenyl)-2-cyano-N'-[1-(4-methoxyphenyl)ethylidene] acrylohydrazide (5c). Yield, 50\%; m.p. 148-150 ${ }^{\circ} \mathrm{C}$ (ethanol); IR (KBr, cm $\left.{ }^{-1}\right)$ : $3313(\mathrm{NH}), 2211(\mathrm{CN}), 1636(\mathrm{CO})$; ${ }^{1} \mathrm{H}$ NMR (DMSO; $\left.\delta \mathrm{ppm}\right): 3.78\left(\mathrm{~s}, 3 \mathrm{H}, \mathrm{OCH}_{3}\right), 2.50(\mathrm{~s}, 3 \mathrm{H}$, $\left.\mathrm{CH}_{3}\right), 6.92-8.06(\mathrm{~m}, 9 \mathrm{H}, \mathrm{Ar}-\mathrm{H}$ and $=\mathrm{CH}$ proton $), 10.06(\mathrm{br}$,
$1 \mathrm{H}, \mathrm{NH}$ ); Anal. for $\mathrm{C}_{19} \mathrm{H}_{16} \mathrm{ClN}_{3} \mathrm{O}_{2}$ (353.80); Calcd.: C, 64.50; $\mathrm{H}, 4.56 ; \mathrm{Cl}, 10.02$; N, 11.88. Found: C, 64.33; H, 4.32; Cl, 10.00; $\mathrm{N}, 11.65 \%$.

2.3.4. 3-(4-Chlorophenyl)- $N^{\prime}$-[(4-chlorophenyl)methylene]-2cyanoacrylohydrazide (5d). Yield, 60\%; m.p. $228-230^{\circ} \mathrm{C}$ (ethanol); IR (KBr, cm ${ }^{-1}$ ): $3282(\mathrm{NH}), 2212(\mathrm{CN}), 1682(\mathrm{CO})$; ${ }^{1} \mathrm{H}$ NMR (DMSO, $\left.\delta \mathrm{ppm}\right): 7.52-8.02(\mathrm{~m}, 9 \mathrm{H}, \mathrm{Ar}-\mathrm{H}$ and $=\mathrm{CH}$ proton $), 8.27(\mathrm{~s}, 1 \mathrm{H},=\mathrm{CH}), 8.71(\mathrm{~s}, 1 \mathrm{H}, \mathrm{NH})$. Anal. for $\mathrm{C}_{17} \mathrm{H}_{11} \mathrm{Cl}_{2} \mathrm{~N}_{3} \mathrm{O}$ (344.19); Calcd.: C, 59.32; H, 3.22; Cl, 20.60; N, 12.21. Found: C, 59.11; H, 3.10; Cl, 20.50; N, 12.01\%.

2.4. General Procedure for Synthesis of Pyrazole-4-carbohydrazide $(\mathbf{6} \boldsymbol{a}, \boldsymbol{b})$. A mixture of $\mathbf{5 a}, \mathbf{b}(0.01)$ and hydrazine hydrate $(0.50 \mathrm{~g}, 0.01 \mathrm{~mol}, 95 \%)$ was refluxed for $6 \mathrm{~h}$. After cooling, the formed solid was filtered, washed with water, and recrystallized from the appropriate solvent to give $\mathbf{6 a}, \mathbf{b}$.

2.4.1. 5-Amino-3-(4-methoxyphenyl)- $N^{\prime}$-[1-(4-methoxyphenyl) ethylidene]-1H-pyrazole-4-carbohydrazide(6a). Yield, 50\%; m.p. $120-124^{\circ} \mathrm{C}$ (ethanol); IR $\left(\mathrm{KBr}, \mathrm{cm}^{-1}\right): 3340,333,3210$ $\left(\mathrm{NH}, \mathrm{NH}_{2}\right), 1663$ (CO); ${ }^{1} \mathrm{H}$ NMR (DMSO, $\left.\delta \mathrm{ppm}\right): 3.82(\mathrm{~s}$, $\left.6 \mathrm{H}, 2 \mathrm{OCH}_{3}\right), 2.56\left(\mathrm{~s}, 3 \mathrm{H}, \mathrm{CH}_{3}\right), 6.99-7.84(\mathrm{~m}, 8 \mathrm{H}, \mathrm{Ar}-\mathrm{H})$, 8.13 (s, 1H, NH), 8.17 (br, 1H, NH), 6.86 (s, 2H, $\mathrm{NH}_{2}$ ). Anal. for $\mathrm{C}_{20} \mathrm{H}_{21} \mathrm{~N}_{5} \mathrm{O}_{3}$ (379.41); Calcd.: C, 63.31; H, 5.58; N, 18.46. Found: C, 63.00; H, 5.32; N, 18.31\%.

2.4.2. 5-Amino-N'-[(4-chlorophenyl)methylene]-3-(4-methoxyphenyl)-1H-pyrazole-4-carbohydrazide (6b). Yield, 55\%; m.p. $140-142^{\circ} \mathrm{C}$ (ethanol); IR $\left(\mathrm{KBr}, \mathrm{cm}^{-1}\right)$ : 3417, 3307, 3287, $\left(\mathrm{NH}, \mathrm{NH}_{2}\right.$ ), 1660 (CO); ${ }^{1} \mathrm{H}$ NMR (DMSO, $\delta$ ppm): 3.74 (s, $\left.3 \mathrm{H}, \mathrm{OCH}_{3}\right), 7.03-7.91(\mathrm{~m}, 9 \mathrm{H}, \mathrm{Ar}-\mathrm{H}$ and $=\mathrm{CH}$ proton $), 8.62$ (s, $1 \mathrm{H}, \mathrm{NH}), 8.71(\mathrm{~s}, 1 \mathrm{H}, \mathrm{NH}), 6.86\left(\mathrm{~s}, 2 \mathrm{H}, \mathrm{NH}_{2}\right) ; \mathrm{MS} \mathrm{m} / z(\%)$ : $369\left(\mathrm{M}^{+}, 1.26\right), 280$ (34.72), 267 (43.68), 134 (100). Anal. for $\mathrm{C}_{18} \mathrm{H}_{16} \mathrm{Cl} \mathrm{N}_{5} \mathrm{O}_{2}$ (369.80); Calcd.: C, 58.46; H, 4.36; Cl, 9.59; N, 18.94. Found: C, 58.22; H, 4.21; Cl, 9.32; N, 18.75\%.

2.5. 2-Amino-4-(4-chlorophenyl)-5-cyano-1-\{[1-(4-methoxyphenyl)ethylidene]amino -6-oxo-1,6-dihydropyridine-3-carboxamide (8). To a solution of compound 3a (2.31 g, $0.01 \mathrm{~mol})$ in 1,4-dioxane $(30 \mathrm{~mL})$ containing few drops of TEA, 3-(4-chlorophenyl)-2-cyanoacrylamide $\quad(2.06 \mathrm{~g}, \quad 0.01 \mathrm{~mol})$ was added. The reaction mixture was heated under reflux for $4 \mathrm{~h}$, then poured into ice/water, and the solid obtained was recrystallized from ethanol to give 8. Yield, 85\%; m.p. 258-260 C; IR (KBr, cm $\left.{ }^{-1}\right): 3435,3313,3210,3129\left(2 \mathrm{NH}_{2}\right)$, 1671, 1660 (2CO), 2211 (CN); ${ }^{1} \mathrm{H}$ NMR (DMSO, $\delta$ ppm): 3.73 (s, 3H, $\left.\mathrm{OCH}_{3}\right), 2.48\left(\mathrm{~s}, 3 \mathrm{H}, \mathrm{CH}_{3}\right), 7.45-7.82(\mathrm{~m}, 8 \mathrm{H}, \mathrm{Ar}-\mathrm{H})$, 6.92 (br, $2 \mathrm{H}, \mathrm{NH}_{2}$ ), 6.95 (br, $2 \mathrm{H}, \mathrm{NH}_{2}$ ); MS m/z (\%): 435 $\left(\mathrm{M}^{+}\right.$, 8.76), 383 (77.70), 282 (14.04). Anal. for $\mathrm{C}_{22} \mathrm{H}_{18} \mathrm{Cl} \mathrm{N}_{5} \mathrm{O}_{3}$ (435.86); Calcd.: C, 60.62; H, 4.16; Cl, 8.13; N, 16.07\%. Found: C, 60.42; H, 4.00; Cl, 8.00; N, 16.00\%.

2.6. 5-(4-Chlorophenyl)-8-\{[1-(4-methoxyphenyl)ethylidene]amino\}-4,7-dioxo-2-(4-nitrophenyl)-3,4,7,8-tetrahydropyrido [2,3-d]pyrimidine-6-carbonitrile (9). A mixture of compound 8 (4.35 g, $0.01 \mathrm{~mol})$ and 4-nitrobenzaldehyde (0.01 mol) in ethanol $(20 \mathrm{~mL})$ with few drops of TEA was refluxed in oil bath with stirring for $10-12 \mathrm{~h}$. The reaction mixture was 
cooled, and poured into ice/water and the solid obtained was recrystallized from ethanol to give 9. Yield, 50\%; m.p. 190-192 ${ }^{\circ} \mathrm{C}$; IR (KBr, cm $\left.{ }^{-1}\right): 3332(\mathrm{NH}), 1660,1675$ (2CO), $2212(\mathrm{CN}) ;{ }^{1} \mathrm{H}$ NMR (DMSO, $\left.\delta \mathrm{ppm}\right): 3.83$ (s, 3H, $\mathrm{OCH}_{3}$ ), 2.50 (s, 3H, $\mathrm{CH}_{3}$ ), 7.46-7.94 (m, 12H, Ar-H), 8.22 (br, $1 \mathrm{H}$, $\mathrm{NH}$ ). Anal. for $\mathrm{C}_{29} \mathrm{H}_{19} \mathrm{ClN}_{6} \mathrm{O}_{5}$ (566.95); Calcd.: C, 61.44; $\mathrm{H}$, 3.38; Cl, 6.25; N, 14.82. Found: C, 61.21; H, 3.08; Cl, 6.00; N, $14.52 \%$.

\subsection{5-(4-Chlorophenyl)-8-\{[1-(4-methoxyphenyl)ethylidene]} amino $\}$-4,7-dioxo-2-thioxo-1,2,3,4,7,8-hexahydropyrido[2,3d]pyrimidine-6-carbonitrile (10). To a stirred solution of $\mathbf{8}$ $(4.35 \mathrm{~g}, 0.01 \mathrm{~mol})$ in $\mathrm{DMF}(10 \mathrm{~mL})$, carbon disulfide $(0.76 \mathrm{~mL}$, $0.01 \mathrm{~mol})$ and $\mathrm{NaOH}(0.2 \mathrm{~g}, 0.005 \mathrm{~mol})$ were added. The reaction mixture was stirred at room temperature for $6 \mathrm{~h}$, then diluted with an equal volume of water, and treated with dilute $\mathrm{HCl}(\mathrm{pH} 4)$. The separated solid product was filtered, washed with water, and recrystallized from DMF to give $\mathbf{1 0 .}$ Yield, 50\%; m.p. $130-132^{\circ} \mathrm{C}$; IR $\left(\mathrm{KBr}, \mathrm{cm}^{-1}\right)$ : 3332, $3188(\mathrm{NH})$, 1660, 1668 (2CO), 2206 (CN), 1243 (C=S); ${ }^{1} \mathrm{H}$ NMR (DMSO, $\delta \mathrm{ppm}): 3.83\left(\mathrm{~s}, 3 \mathrm{H}, \mathrm{OCH}_{3}\right), 2.51\left(\mathrm{~s}, 3 \mathrm{H}, \mathrm{CH}_{3}\right), 6.93-7.60$ (m, 8H, Ar-H), 8.62 (br, 1H, NH), 8.50 (br, 1H, NH); MS $\mathrm{m} / z$ (\%): $478\left(\mathrm{M}^{+}+1,1.58\right), 270$ (12.99), 229 (17.15), 143 (100). Anal. for $\mathrm{C}_{23} \mathrm{H}_{16} \mathrm{ClN}_{5} \mathrm{O}_{3} \mathrm{~S}$ (477.92); Calcd.: C, 57.80; H, 3.37; $\mathrm{Cl}, 7.42$; N, 14.65; S, 6.71. Found: C, 57.50; H, 3.30; Cl, 7.25; N, 14.38; S, $6.52 \%$.

2.8. General Procedure for Synthesis of 4,5,6,7-Tetrahydrobenzo[b]thiophene-2-carbohydrazide Derivatives $(\mathbf{1 1 a}, \boldsymbol{b})$. To a solution of $3 \mathbf{a}, \mathbf{b}(0.01 \mathrm{~mol})$ in ethanol $(20 \mathrm{~mL})$ containing TEA $(1 \mathrm{~mL})$ and elemental sulfur $(0.01 \mathrm{~mol})$, cyclohexanone $(0.01 \mathrm{~mol})$ was added. The reaction mixture was heated under reflux for $3 \mathrm{~h}$, then poured into ice/water. The formed solid product was collected by filtration and recrystallized from the appropriate solvent to give 11a,b.

2.8.1. 3-Amino- $N^{\prime}$-[1-(4-methoxyphenyl)ethylidene]-4,5,6,7tetrahydrobenzo[b] thiophene-2-carbohydrazide (11a). Yield; $70 \%$; m.p. $118-120^{\circ} \mathrm{C}$ (pot.ether 60-80); IR $\left(\mathrm{KBr}, \mathrm{cm}^{-1}\right)$ : 3391, 3298, $3267\left(\mathrm{NH}, \mathrm{NH}_{2}\right), 1698(\mathrm{CO}) ;{ }^{1} \mathrm{H}$ NMR (DMSO, $\delta \mathrm{ppm}): 3.80\left(\mathrm{~s}, 3 \mathrm{H}, \mathrm{OCH}_{3}\right), 2.51\left(\mathrm{~s}, 3 \mathrm{H}, \mathrm{CH}_{3}\right), 6.94-7.84(\mathrm{~m}$, $4 \mathrm{H}, \mathrm{Ar}-\mathrm{H}), 6.40$ (br, 2H, NH $\left.\mathrm{NH}_{2}\right), 10.40$ (br, 1H, NH), 1.71-1.73 $\left(\mathrm{m}, 4 \mathrm{H}, 2 \mathrm{CH}_{2}\right), 2.19-2.44\left(\mathrm{~m}, 4 \mathrm{H}, 2 \mathrm{CH}_{2}\right)$. Anal. for $\mathrm{C}_{18} \mathrm{H}_{21}$ $\mathrm{N}_{3} \mathrm{O}_{2} \mathrm{~S}$ (343.44); Calcd.: C, 62.95; H, 6.16; N, 12.23; S, 9.34. Found: C, 62.65; H, 6.00; N, 12.00; S, 9.11\%.

2.8.2. 3-Amino- $N^{\prime}$-[(4-chlorophenyl)methylene]-4,5,6,7-tetrahydrobenzo[b] thiophene-2-carbohydrazide (11b). Yield, 85\%; m.p. $110-112^{\circ} \mathrm{C}$ (ethanol); IR $\left(\mathrm{KBr}, \mathrm{cm}^{-1}\right), 3425,3298,3267$ $\left(\mathrm{NH}, \mathrm{NH}_{2}\right), 1624(\mathrm{CO}) ;{ }^{1} \mathrm{H}$ NMR (DMSO, $\delta$ ppm): 7.30-7.41 $(\mathrm{m}, 5 \mathrm{H}, \mathrm{Ar}-\mathrm{H}$ and $=\mathrm{CH}), 4.30\left(\right.$ br, $\left.2 \mathrm{H}, \mathrm{NH}_{2}\right), 8.42(\mathrm{br}, 1 \mathrm{H}$, $\mathrm{NH}), 2.48-2.50\left(\mathrm{~m}, 8 \mathrm{H}, 4 \mathrm{CH}_{2}\right)$. Anal. for $\mathrm{C}_{16} \mathrm{H}_{16} \mathrm{Cl} \mathrm{N}_{3} \mathrm{OS}$ (333.83); Calcd.: C, 57.56; H, 4.83; Cl, 10.62; N, 12.59; S, 9.61. Found: C, 57.32; H, 4.52; Cl, 10.42; N, 12.41; S, 9.32\%.

2.9. 2-Cyano- $N^{\prime}$-[1-(4-methoxyphenyl)ethylidene]-2-(4-oxo-3phenyl-1,3-thiazolidin-2-ylidene)ethanehydrazide (14). To a stirred suspension of finely powdered potassium hydroxide $(0.01 \mathrm{~mole})$ in dry DMF $(10 \mathrm{~mL})$, cyanoacetohydrazide (3a) (2.31 g, 0.01 mole) was added and continuous stirring was done for $30 \mathrm{~min}$. Then phenylisothiocyanate $(1.35 \mathrm{~mL}$, $0.01 \mathrm{~mol}$ ) was added slowly over the course of $10 \mathrm{~min}$. After complete addition, stirring of the reaction mixture was done at room temperature for $12 \mathrm{~h}$. Then ethyl chloroacetate $(1.22 \mathrm{~mL}, 0.01 \mathrm{~mole})$ was added to the reaction mixture and stirred for $6 \mathrm{~h}$. The reaction mixture was poured into crushed ice. The resulting precipitate was filtrated off, dried, and recrystallized from ethanol/DMF to give 14. Yield, 85\%; m.p. 264-266 ${ }^{\circ}$; IR (KBr, cm ${ }^{-1}$ ), $3370(\mathrm{NH}), 1742,1667$ (2CO), $2184(\mathrm{CN}) ;{ }^{1} \mathrm{H}$ NMR (DMSO, $\left.\delta \mathrm{ppm}\right): 3.79$ (s, 3H, $\mathrm{OCH}_{3}$ ), 2.43 (s, 3H, $\mathrm{CH}_{3}$ ), 4.02 (s, 2H, C 4-2H-thiazolidinone), 6.947.76 (m, 9H, Ar-H), 9.83 (br, $1 \mathrm{H}, \mathrm{NH})$; MS m/z (\%): $406\left(\mathrm{M}^{+}\right.$, 41.28), 391 (13.87), 360 (11.96), 314 (10.32), 281 (33.41), 134 (100). Anal. for $\mathrm{C}_{21} \mathrm{H}_{18} \mathrm{~N}_{4} \mathrm{O}_{3} \mathrm{~S}$ (406.45); Calcd.: C, 62.05; H, 4.46; N, 13.78; S, 7.89. Found: C, 61.88; H, 4.22; N, 13.62; S, $7.55 \%$.

2.10. 3-Anilino-2-cyano- $N^{\prime}$-[1-(4-methoxyphenyl)ethylidene]3-methylthio-acrylohydrazide (15). Compound 15 was synthesized as mentioned for the synthesis of $\mathbf{1 4}$ but using dimethylsulfate instead of ethylchloroacetate; the resulting product was crystallized from ethanol to give 15 .

Yield, 70\%, m.p. $156-158^{\circ} \mathrm{C}$; IR (KBr, cm $\left.{ }^{-1}\right), 3374,3318$ (2NH), 1642 (CO), $2182(\mathrm{CN}) ;{ }^{1} \mathrm{H}$ NMR (DMSO, $\delta$ ppm): 3.81 $\left(\mathrm{s}, 3 \mathrm{H}, \mathrm{OCH}_{3}\right), 2.33\left(\mathrm{~s}, 3 \mathrm{H}, \mathrm{CH}_{3}\right), 2.51\left(\mathrm{~s}, 3 \mathrm{H}, \mathrm{SCH}_{3}\right), 6.94-7.82$ (m, 9H, Ar-H), 10.11 (br, 1H, NH Ph), 11.00 (br, 1H, NH); MS $m / z(\%): 380\left(\mathrm{M}^{+}, 25.28\right), 333$ (53.86), 365 (1.83), 318 (7.81), 231 (2.55), 217 (40.63), 163 (55.56). Anal. for $\mathrm{C}_{20} \mathrm{H}_{20} \mathrm{~N}_{4} \mathrm{O}_{2} \mathrm{~S}$ (380.46); Calcd.: C, 63.14; H, 5.30; N, 14.73; S, 8.43. Found: C, $63.00 ; \mathrm{H}, 5.00 ; \mathrm{N}, 14.52 ; \mathrm{S}, 8.30 \%$.

2.11. 5-Amino-3-anilino- $N^{\prime}$-[1-(4-methoxyphenyl)ethylidene]1H-pyrazole-4-carbohydrazide (16). A mixture of 15 (3.80 g, $0.01 \mathrm{~mol})$ and hydrazine hydrate $(0.012 \mathrm{~mol})$ in ethanol $(30 \mathrm{~mL})$ was heated under reflux for $3 \mathrm{~h}$. and allowed to cool. The solid product obtained was filtered and recrystallized from ethanol to give 16. Yield, $55 \%$; m.p. $122-125^{\circ} \mathrm{C}$; IR $(\mathrm{KBr}$, $\left.\mathrm{cm}^{-1}\right)$ : 3391, 3298, $3242\left(\mathrm{NH}, \mathrm{NH}_{2}\right), 1641(\mathrm{CO}) ;{ }^{1} \mathrm{H}$ NMR (DMSO, $\delta \mathrm{ppm}): 3.77$ (s, 3H, $\mathrm{OCH}_{3}$ ), 2.27 (s, 3H, $\mathrm{CH}_{3}$ ), 6.857.57 (m, 9H, Ar-H), 6.13 (s, 2H, $\mathrm{NH}_{2}$ ), 8.00 (br, $1 \mathrm{H}, \mathrm{NH} \mathrm{Ph),}$ 8.20 (br, 1H, NHCO), 10.10 (br, $1 \mathrm{H}, \mathrm{NH}$ ); MS $m / z$ (\%): 364 $\left(\mathrm{M}^{+}, 0.41\right), 348$ (0.47), 318 (2.92), 281 (28.52), 267 (22.88), 256 (0.71), 201 (1.60), 148 (47.34), 134 (100). Anal. for $\mathrm{C}_{19} \mathrm{H}_{20} \mathrm{~N}_{6} \mathrm{O}_{2}$ (364.40); Calcd: C, 62.62; H, 5.53; N, 23.06. Found: C, 62.32; H, 5.23; N, 23.00\%.

2.12. General Procedure for Synthesis of 3,3-Dimercaptoacrylohydrazide Derivatives $(\mathbf{1 8 a}, \boldsymbol{b})$. To suspension of finely powdered potassium hydroxide $(0.04 \mathrm{~mol})$ in dry DMF $(20 \mathrm{~mL})$ at $0^{\circ} \mathrm{C}$ the cyanoacetohydrazide $3 \mathbf{a}, \mathbf{b}(0.04 \mathrm{~mol})$ was added for $30 \mathrm{~min}$, carbon disulfide $(3.04 \mathrm{~mL}, 0.04 \mathrm{~mol})$ was added to the resulting mixture, stirring was continued for $12 \mathrm{~h}$, and then hydrochloric acid (2 M, $20 \mathrm{~mL}$ was added dropwise, and stirring continued for additional $1 \mathrm{~h}$. Then, the reaction mixture was poured into ice water. The solid product that formed was filtered off, dried, and recrystallized from the appropriate solvent to give $\mathbf{1 8 a}, \mathbf{b}$. 
2.12.1. 2-Cyano-3,3-dimercapto- $N^{\prime}$-[1-(4-methoxyphenyl)ethylidene]acrylohydrazide (18a). Yield, 70\%; m.p. 248-250 ${ }^{\circ} \mathrm{C}$ (ethanol); IR (KBr, cm ${ }^{-1}$ ): 3225 (NH), 1663 (CO); ${ }^{1} \mathrm{H}$ NMR (DMSO, $\delta$ ppm): 3.7 (s, 3H, $\mathrm{OCH}_{3}$ ), 2.49 (s, 3H, $\mathrm{CH}_{3}$ ), 6.977.95 (m, 4H, Ar-H), 10.26 (br, 1H, NHCO), 2.73 (s, 2H, 2SH); MS $m / z$ (\%): $306\left(\mathrm{M}^{+}-1,3.66\right), 281$ (1.93), 274 (8.92), 241 (5.42), 215 (6.25), 163 (22.05), 134 (100). Anal. for $\mathrm{C}_{13} \mathrm{H}_{13}$ $\mathrm{N}_{3} \mathrm{O}_{2} \mathrm{~S}_{2}$ (307.39); Calcd.: C, 50.79; H, 4.26; N, 13.67; S, $20.86 \%$. Found: C, 50.45; H, 4.00; N, 13.32; S, 20.52\%.

2.12.2. $N^{\prime}$-[(4-Chlorophenyl)methylene]-2-cyano-3,3-dimercaptoacrylohydrazide (18b). Yield: 75\%; m.p. $190-192^{\circ} \mathrm{C}$ (ethanol); IR (KBr, cm ${ }^{-1}$ ): $3333(\mathrm{NH}), 1673(\mathrm{CO}) ;{ }^{1} \mathrm{H}$ NMR (DMSO, $\delta$ ppm): $8.16(\mathrm{~s}, 1 \mathrm{H}, \mathrm{CH}=\mathrm{N}), 7.48-7.99(\mathrm{~m}, 4 \mathrm{H}$, Ar-H), 11.82 (br, 1H, NH CO), 2.48 (s, 2H, 2SH). MS m/z (\%): 297 ( $\left.\mathrm{M}^{+}, 4.75\right), 271$ (3.86), 264 (4.55), 231 (3.45), 205 (4.63), 161 (26.97), 158 (4.02), 153 (16.19), 143 (11.75), 138 (100), 115 (8.28), 78 (51.02). Anal. for $\mathrm{C}_{11} \mathrm{H}_{8} \mathrm{ClN}_{3} \mathrm{OS}_{2}$ (297.78); Calcd.: C, 44.37; H, 2.71; Cl, 11.91; N, 14.11; S, 21.54\%. Found: C, 44.00; H, 2.32; Cl, 11.66; N, 14.00; S, 21.22\%.

2.13. General Procedure for Synthesis of Ethyl [2-Cyano-1mercapto-3-oxoprop-1-enyl)thio]acetate Derivatives $(\mathbf{1 9 a}, \boldsymbol{b})$. To a stirred suspension of finely powdered potassium hydroxide $(0.02$ mole) in dry DMF $(10 \mathrm{~mL})$, cyanoacetohydrazides, 3a,b, (0.01 mole) were added. The resulted mixture was cooled at $10^{\circ} \mathrm{C}$ in an ice bath; then $(0.01 \mathrm{~mol})$ carbon disulfide was added slowly over the course of $10 \mathrm{~min}$. After complete addition, stirring of the reaction mixture was continued for $6 \mathrm{~h}$ Then ethyl chloroacetate $(1.22,0.01 \mathrm{~mol})$ was added to the mixture and stirring continued for $3 \mathrm{~h}$, then the mixture was poured into crushed ice and $\mathrm{HCl}$; the resulting precipitate was filtrated off, dried, and recrystallized from the appropriate solvent to give $\mathbf{1 9 a}, \mathbf{b}$.

2.13.1. Ethyl [2-Cyano-1-mercapto-3-\{2-[1-(4-methoxyphenyl) ethylidene]hydrazino\}-3-oxoprop-1-enyl)thio]acetate (19a). Yield, 70\%; m.p. $236-240^{\circ} \mathrm{C}$ (ethanol); IR (KBr, $\mathrm{cm}^{-1}$ ): 3331 $(\mathrm{NH}) ; 2216(\mathrm{CN}) ;{ }^{1} \mathrm{H}$ NMR (DMSO $\left.\delta \mathrm{ppm}\right): 3.84(\mathrm{~s}, 3 \mathrm{H}$, $\left.\mathrm{OCH}_{3}\right), 2.51\left(\mathrm{~s}, 3 \mathrm{H}, \mathrm{CH}_{3}\right), 7.02-7.94(\mathrm{~m}, 4 \mathrm{H}, \mathrm{Ar}-\mathrm{H}), 11.28$ (br, $1 \mathrm{H}, \mathrm{NH}), 11.54$ (br, $1 \mathrm{H}, \mathrm{SH}), 1.03$ (t, 3H, $\mathrm{CH}_{3}$ ), 4.33 (s, 2H, $\mathrm{CH}_{2}$ ), 4.12 (q, 2H, $\mathrm{CH}_{2}$ ). Anal. for $\mathrm{C}_{17} \mathrm{H}_{19} \mathrm{~N}_{3} \mathrm{O}_{4} \mathrm{~S}_{2}$ (393.48); Calcd.: C, 51.86; H, 4.87; N, 10.68; S, 16.30. Found: C, 51.53; $\mathrm{H}, 4.52 ; \mathrm{N}, 10.51 ; \mathrm{S}, 16.00 \%$.

2.13.2.Ethyl (\{3-[2-(4-Chlorobenzylidene)hydrazino]-2-cyano1-mercapto-3-oxoprop-1-enyl\}thio)acetate (19b). Yield, 65\%; m.p. $148-150^{\circ} \mathrm{C}$ (ethanol); IR $\left(\mathrm{KBr}, \mathrm{cm}^{-1}\right): 3359(\mathrm{NH}), 2360$ $(\mathrm{CN}) ;{ }^{1} \mathrm{H}$ NMR (DMSO; $\left.\delta \mathrm{ppm}\right): 7.38-7.65$ (m, 4H, Ar-H), $8.27(\mathrm{~s}, 1 \mathrm{H}, \mathrm{CH}=\mathrm{C}), 1.05\left(\mathrm{t}, 3 \mathrm{H}, \mathrm{CH}_{3}\right), 4.22\left(\mathrm{q}, 2 \mathrm{H}, \mathrm{CH}_{2}\right), 4.02$ (s, 2H, $\mathrm{CH}_{2}$ ), 9.07 (br, $\left.1 \mathrm{H}, \mathrm{NH}\right), 10.02$ (br, $1 \mathrm{H}, \mathrm{SH}$ ). Anal. for $\mathrm{C}_{15} \mathrm{H}_{14} \mathrm{Cl} \mathrm{N}_{3} \mathrm{O}_{3} \mathrm{~S}_{2}$ (383.87); Calcd.: C, 46.93; H, 3.68; Cl, 9.24; N, 10.95; S, 16.71. Found: C, 46.73; H, 3.34; Cl, 9.00; N, 10.65; S, $16.45 \%$.

2.14. General Procedure for Synthesis of 3,3-Bis(methylthio) acrylohydrazide Derivatives $(\mathbf{2 0 a}, \boldsymbol{b})$. Compounds 20a,b were synthesized as mentioned for synthesis of 19, but using dimethyl sulfate instead of ethyl chloroacetate, the resulting product was recrystallized from the appropriate solvent to give 20a,b.

2.14.1. 2-Cyano- $N^{\prime}$-[(1-(4-methoxyphenyl)ethylidene]-3,3-bis(methylthio)-acrylohydrazide (20a). Yield, 70\%; m.p. 168$170^{\circ} \mathrm{C}$ (pot.ether $60-80 /$ benzene): IR $\left(\mathrm{KBr}, \mathrm{cm}^{-1}\right), 3203(\mathrm{NH})$, 1681 (CO); ${ }^{1} \mathrm{H}$ NMR (DMSO, $\left.\delta \mathrm{ppm}\right): 3.78$ (s, $3 \mathrm{H}, \mathrm{OCH}_{3}$ ), $2.49\left(\mathrm{~s}, 3 \mathrm{H}, \mathrm{CH}_{3}\right), 2.50\left(\mathrm{~s}, 6 \mathrm{H}, 2 \mathrm{SCH}_{3}\right), 6.93-7.76(\mathrm{~m}, 4 \mathrm{H}, \mathrm{Ar}-$ $\mathrm{H}), 10.90$ (br, $1 \mathrm{H}, \mathrm{NH} \mathrm{CO}$ ). Anal. for $\mathrm{C}_{15} \mathrm{H}_{17} \mathrm{~N}_{3} \mathrm{O}_{2} \mathrm{~S}_{2}$ (335.44); Calcd.: C, 53.71; H, 5.11; N, 12.53; S, 19.12. Found: C, 53.52; H, $5.00 ; \mathrm{N}, 12.23 ; \mathrm{S}, 19.00 \%$.

2.14.2. $N^{\prime}$-[(4-Chlorophenyl)methylene]-2-cyano-3,3-bis(methylthio)acrylohydrazide (20b). Yield, 75\%; m.p. $188-190^{\circ} \mathrm{C}$ (ethanol); IR (KBr, cm ${ }^{-1}$ ), $3185(\mathrm{NH}), 1674(\mathrm{CO}) ;{ }^{1} \mathrm{H}$ NMR (DMSO, $\delta$ ppm): $8.16(\mathrm{~s}, 1 \mathrm{H}, \mathrm{CH}=\mathrm{N}), 7.48-7.99(\mathrm{~m}, 4 \mathrm{H}, \mathrm{Ar}-$ $\mathrm{H}), 11.82$ (br, $1 \mathrm{H}, \mathrm{NH} \mathrm{CO}), 2.50\left(\mathrm{~s}, 6 \mathrm{H}, 2 \mathrm{SCH}_{3}\right) ; \mathrm{MS} \mathrm{m} / z(\%)$ : $325\left(\mathrm{M}^{+}, 10.74\right), 295$ (17.07), 153 (5.49), 138 (100). Anal. for $\mathrm{C}_{13} \mathrm{H}_{12} \mathrm{ClN}_{3} \mathrm{OS}_{2}$ (325.83); Calcd.: C, 47.92; H, 3.71; Cl, 10.88; N, 12.90; S, 19.68. Found: C, 47.64; H, 3.42; Cl, 10.55; N, 12.65; S, $19.48 \%$.

2.15. General Procedure for Synthesis of 5-Amino-3-methylthiopyrazole-4-carbohydrazide (21 and 22). A mixture of 20a (3.35 g, $0.01 \mathrm{~mol})$, hydrazine hydrate, and/or 2-hydrazino1,3-benzothiazole $(1.89 \mathrm{~g}, 0.012 \mathrm{~mol})$ in ethanol $(30 \mathrm{~mL})$ was heated under reflux for $3 \mathrm{hrs}$ and allowed to cool. The solid product obtained was filtrate off and recrystallized from the appropriate solvent to give $\mathbf{2 1}$ and $\mathbf{2 2}$.

2.15.1. 5-Amino-N'-[1-(4-methoxyphenyl)ethylidene]-3-methylthio-pyrazole-4-carbohydrazide (21). Yield, 65\%; m.p. 120$122^{\circ} \mathrm{C}$ (ethanol); IR $\left(\mathrm{KBr}, \mathrm{cm}^{-1}\right): 3392,3300,3243\left(\mathrm{NH}, \mathrm{NH}_{2}\right)$; ${ }^{1} \mathrm{H}$ NMR (DMSO, $\left.\delta \mathrm{ppm}\right): 3.74\left(\mathrm{~s}, 3 \mathrm{H}, \mathrm{OCH}_{3}\right), 2.27(\mathrm{~s}, 3 \mathrm{H}$, $\mathrm{CH}_{3}$ ), 2.50 (s, 3H, $\mathrm{SCH}_{3}$ ), 6.85-7.56 (m, 4H, Ar-H), 6.13 (br, $2 \mathrm{H}, \mathrm{NH}_{2}$ ), 8.00 (br, $\left.1 \mathrm{H}, \mathrm{NH}\right), 10.10$ (br, $\left.1 \mathrm{H}, \mathrm{NH}\right) ; \mathrm{MS} m / z(\%)$ : $319\left(\mathrm{M}^{+}, 0.81\right), 275$ (1.24), 259 (1.44), 246 (4.66), 228 (4.80), 210 (3.20), 176 (16.37), 162 (44.23), 128 (24.43), 134 (100). Anal. for $\mathrm{C}_{14} \mathrm{H}_{17} \mathrm{~N}_{5} \mathrm{O}_{2} \mathrm{~S}$ (319.38); Calcd.: C, 52.65; H, 5.37; N, 21.93; S, 10.04. Found: C, 52.42; H, 5.21; N, 21.73; S, 10.00\%.

2.15.2. 5-Amino-1-(1,3-benzothiazol-2-yl)- $N^{\prime}$-[1-(4-methoxyphenyl)ethylidene]-3-methylthio-pyrazole-4-carbohydrazide (22). Yield, 70\%; m.p. $150-152^{\circ} \mathrm{C}$ (acetone); IR $\left(\mathrm{KBr}, \mathrm{cm}^{-1}\right)$ : 3392, 3300, $3243\left(\mathrm{NH}, \mathrm{NH}_{2}\right) ;{ }^{1} \mathrm{H}-\mathrm{NMR}$ (DMSO, $\left.\delta \mathrm{ppm}\right)$ : $3.76\left(\mathrm{~s}, 3 \mathrm{H}, \mathrm{OCH}_{3}\right), 2.23\left(\mathrm{~s}, 3 \mathrm{H}, \mathrm{CH}_{3}\right), 2.50\left(\mathrm{~s}, 3 \mathrm{H}, \mathrm{SCH}_{3}\right)$, 6.85-7.56 (m, 8H, Ar-H), $6.13\left(\mathrm{~s}, 2 \mathrm{H}, \mathrm{NH}_{2}\right), 10.44$ (br, $1 \mathrm{H}$, $\mathrm{NH}), 453\left(\mathrm{M}^{+}+1,16.51\right), 420$ (12.55), 342 (5.93), 267 (2.54), 253 (4.46), 235 (100), 237 (8.19). Anal. for $\mathrm{C}_{21} \mathrm{H}_{20} \mathrm{~N}_{6} \mathrm{O}_{2} \mathrm{~S}_{2}$ (452.55); Calcd.: C, 55.73; H, 4.45; N, 18.57; S, 14.17. Found: C, 55.52; H, 4.12; N, 18.23; S, $14.00 \%$.

2.16. Antitumor Activity. Antitumor activity was performed in Regional Center for Mycology and Biotechnology, AlAzhar University, Cairo, Egypt. Compounds 3a, 3b, 4a, 4b, 
TABLE 1: In vitro anticancer screening of the synthesized compounds against human breast cancer cell line (MCF-7) at different concentration.

\begin{tabular}{|c|c|c|c|c|c|c|c|}
\hline \multirow{2}{*}{ Compd. no. } & \multicolumn{7}{|c|}{ Compound concentrations $(\mu \mathrm{g} / \mathrm{mL})$} \\
\hline & $1.56(\mu \mathrm{g} / \mathrm{mL})$ & $3.125(\mu \mathrm{g} / \mathrm{mL})$ & $6.25(\mu \mathrm{g} / \mathrm{mL})$ & $12.5(\mu \mathrm{g} / \mathrm{mL})$ & $25(\mu \mathrm{g} / \mathrm{mL})$ & $50(\mu \mathrm{g} / \mathrm{mL})$ & $\mathrm{IC50}(\mu \mathrm{g} / \mathrm{mL})$ \\
\hline doxorubicin & 30.86 & 21.18 & 17.22 & 11.74 & 6.55 & 3.24 & 0.426 \\
\hline $3 a$ & 94.28 & 85.92 & 73.36 & 98.63 & 47.25 & 14.94 & 23.4 \\
\hline $3 \mathbf{b}$ & 94.76 & 89.14 & 80.92 & 67.48 & 54.3 & 22.38 & 28.4 \\
\hline $4 a$ & 95.08 & 88.93 & 79.34 & 67.68 & 47.92 & 16.58 & 23.7 \\
\hline $4 b$ & 98.48 & 93.07 & 87.12 & 72.35 & 51.94 & 27.19 & 27 \\
\hline $6 a$ & 97.32 & 86.46 & 77.95 & 48.52 & 27.04 & 10.71 & 12.2 \\
\hline $6 b$ & 100 & 97.68 & 89.73 & 73.48 & 51.42 & 19.17 & 26.1 \\
\hline $11 b$ & 84.58 & 69.75 & 56.84 & 23.08 & 19.32 & 10.58 & 7.5 \\
\hline 14 & 100 & 98.17 & 91.98 & 86.3 & 63.62 & 29.56 & 35 \\
\hline 15 & 100 & 96.54 & 88.72 & 73.91 & 60.58 & 28.92 & 33.4 \\
\hline 16 & 91.96 & 82.55 & 71.67 & 50.11 & 41.36 & 29.18 & 12.6 \\
\hline $18 b$ & 96.98 & 93.14 & 76.92 & 68.45 & 59.73 & 31.08 & 33.5 \\
\hline 19a & 100 & 98.42 & 93.16 & 90.34 & 78.19 & 61.92 & 50 \\
\hline $19 b$ & 89.34 & 81.9 & 69.79 & 53.41 & 38.18 & 26.87 & 15.3 \\
\hline 21 & 93.81 & 88.94 & 79.22 & 63.75 & 16.28 & 9.64 & 16.1 \\
\hline 22 & 80.36 & 69.42 & 61.87 & 56.39 & 41.81 & 18.26 & 18 \\
\hline
\end{tabular}

IC50 value: corresponds to the concentration required for $50 \%$ inhibition of cell viability.

$6 a, 6 b, 11 b, 14,15,16,18 b, 19 a, 19 b, 21$, and 22 were tested for their cytotoxicity in vitro, in comparison with doxorubicin (DXR) as a reference drug against human breast cancer cell line $\left(\right.$ MCF-7). MCF-7 cells $\left(1 \times 10^{4}\right)$ were incubated with synthesized compounds at various concentrations of 0.39 , $0.78,1.56,3.125,6.25,12.5,25$, and $50 \mu \mathrm{g} / \mathrm{mL}$ (incorporated with $10 \mu \mathrm{L}$ DMSO) at $37^{\circ} \mathrm{C}$ for $48 \mathrm{~h}$, and viable cells yield was determined by colorimetric method [11]. Experiments were carried out in triplicate, and results are reported in Table 1.

\section{Results and Discussion}

3.1. Synthesis. The Schemes 1-4 describe the synthesis of the target molecules. Condensation of cyanoacetic acid hydrazide (1) with 4-methoxyacetophenone and 4-chlorobenzaldehyde(2a,b) in hot 1,4-dioxane afforded hydrazone derivatives $(\mathbf{3 a}, \mathbf{b})$. The structures of compounds (3a,b) were established on the basis of analytical and spectral data. IR spectrum of $3 \mathbf{a}$ showed bands at $3204 \mathrm{~cm}^{-1}$ (NH), 2256 $(\mathrm{CN})$, and $1675(\mathrm{C}=\mathrm{O}) .{ }^{1} \mathrm{H}$ NMR spectrum of $\mathbf{3 a}$ showed the presence of a singlet at $\delta 2.23 \mathrm{ppm}$ corresponding to $\mathrm{CH}_{3}$ group, a singlet at $\delta 3.81 \mathrm{ppm}$ for methoxy group, a singlet at $4.18 \mathrm{ppm}$ for $\mathrm{CH}_{2}$ group, and a singlet at $\delta 10.90 \mathrm{ppm}$ for an $\mathrm{NH}$ group. Further evidence for the structures of $\mathbf{3 a}, \mathbf{b}$ was obtained through studying their chemical reactivity through some chemical reagents. Thus, cyclocondensation of compounds 3a,b with salicylaldehyde in boiling ethanol and in the presence of a few drops of triethylamine (TEA) [12] afforded the corresponding $2 \mathrm{H}$-chromene-3-carbohydrazide derivatives, $\mathbf{4 a}, \mathbf{b}$.

Condensed 3a,b with aryl aldehydes such as 4-methoxybenzaldehyde and/or 4-chlorobenzaldehyde [13] afforded the corresponding arylidene derivatives $\mathbf{5} \mathbf{a}-\mathbf{d}$. The structures of compounds $5 \mathbf{a}-\mathbf{d}$ were based on analytical and spectral data (see Section 2). When compounds 5a,b reacted with hydrazine hydrate [14], they afforded the corresponding $1 \mathrm{H}$ pyrazole-4-carbohydrazide derivatives, $\mathbf{6 a}, \mathbf{b}$. The structure of 6 was inferred from its ${ }^{1} \mathrm{H}$ NMR spectrum which appeared as new signals for $\mathrm{NH}_{2}$ group (Scheme 1).

Reactivity of hydrazide-hydrazone derivative 3a toward cinnamonitrile derivative was studied [15]. Thus, the reaction of 3a with 3-(4-chlorophenyl)-2-cyanoacrylamide afforded the corresponding pyridinone derivative $\mathbf{8}$; the reaction took place through the intermediate 7 . Treatment of pyridinone derivative $\mathbf{8}$ with 4 -nitrobenzaldehyde in the presence of ethanol and catalytic amount of triethylamine (TEA) afforded the corresponding pyrido[2,3-d]pyrimidine6-carbonitrile derivative 9. On the other hand, 2-thioxopyrido[2,3-d]pyrimidine-6-carbonitrile $\mathbf{1 0}$ was obtained by cyclizing $\mathbf{8}$ with carbon disulfide in the presence of sodium hydroxide at room temperature [16] followed by acidification with diluted hydrochloric acid. Structures of new compounds were based on analytical and spectral data. Thus, the ${ }^{1} \mathrm{H}$ NMR spectrum of $\mathbf{8}$ showed the presence of a singlet at $\delta 6.92$ and 6.95 corresponding to the two $\mathrm{NH}_{2}$ groups. The IR spectrum of 10 displayed absorption bands at $3332,3188 \mathrm{~cm}^{-1}$ due to the $2 \mathrm{NH}$ function and $1243 \mathrm{~cm}^{-1}$ due to $\mathrm{C}=\mathrm{S}$ group.

Reaction of compounds $\mathbf{3 a}, \mathbf{b}$ with cyclohexanone and elemental sulfur in the presence of TEA afforded the corresponding 4,5,6,7-tetrahydrobenzo[b] thiophene derivatives, 11a,b. Formation of $\mathbf{1 1}$ took place according to the similar reported reactions of cyclohexanone with methylene reagents and elemental sulfur [17]. Structure of compound $\mathbf{1 1}$ was based on analytical and spectral data (Scheme 2$)$.

The active methylene moiety of 2-cyano- $\mathrm{N}^{\prime}$-[1-(4-methoxyphenyl)ethylidene]acetohydrazide (3a) allowed reacting with phenylisothiocyanate in dry $N, N$-dimethylformamide DMF containing catalytic amount of potassium hydroxide 


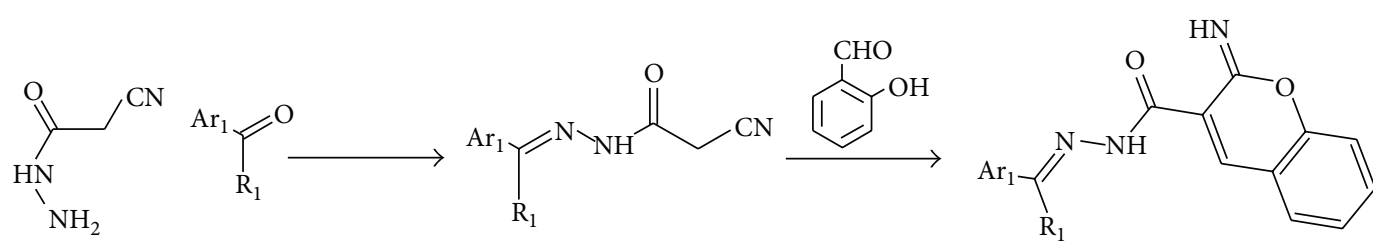

1

2 3

\begin{tabular}{|c|c|c|c|c|c|}
\hline 2 and 3 & $\mathrm{Ar}_{1}$ & $\mathrm{R}_{1}$ & & $4 \mathbf{a} ; \mathrm{Ar}_{1}=p-\mathrm{CH}_{3} \mathrm{OPh}$ & $\mathrm{R}_{1}=\mathrm{CH}_{3}$ \\
\hline $\mathbf{a}$ & $p-\mathrm{CH}_{3} \mathrm{OPh}$ & $\mathrm{CH}_{3}$ & $\mathrm{Ar}_{2} \mathrm{CHO}$ & $\mathbf{b} ; \mathrm{Ar}_{1}=p-\mathrm{ClPh}$ & $\mathrm{R}_{1}=\mathrm{H}$ \\
\hline
\end{tabular}

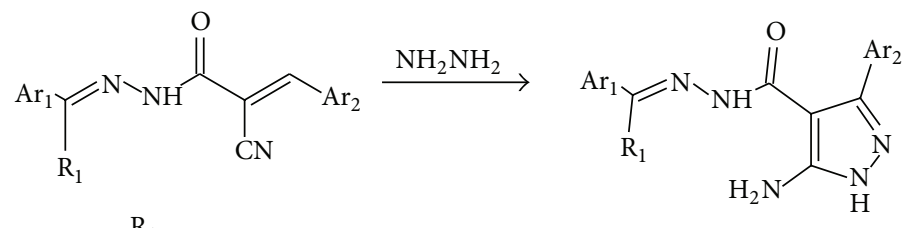

\begin{tabular}{c|ccc}
$\mathbf{5}$ & $\mathrm{Ar}_{1}$ & $\mathrm{Ar}_{2}$ & $\mathrm{R}_{1}$ \\
\hline $\mathbf{a}$ & $p-\mathrm{CH}_{3} \mathrm{OPh}$ & $p-\mathrm{CH}_{3} \mathrm{OPh}$ & $\mathrm{CH}_{3}$ \\
$\mathbf{b}$ & $p-\mathrm{ClPh}$ & $p-\mathrm{CH}_{3} \mathrm{OPh}$ & $\mathrm{H}$ \\
$\mathbf{c}$ & $p-\mathrm{CH}_{3} \mathrm{OPh}$ & $p-\mathrm{ClPh}$ & $\mathrm{CH}_{3}$ \\
$\mathbf{d}$ & $p-\mathrm{ClPh}$ & $p-\mathrm{ClPh}$ & $\mathrm{H}$
\end{tabular}

\begin{tabular}{c|ccc}
$\mathbf{6}$ & $\mathrm{Ar}_{1}$ & $\mathrm{Ar}_{2}$ & $\mathrm{R}_{1}$ \\
\hline $\mathbf{a}$ & $p-\mathrm{CH}_{3} \mathrm{OPh}$ & $p-\mathrm{CH}_{3} \mathrm{OPh}$ & $\mathrm{CH}_{3}$ \\
$\mathbf{b}$ & $p-\mathrm{ClPh}$ & $p-\mathrm{CH}_{3} \mathrm{OPh}$ & $\mathrm{H}$
\end{tabular}

Scheme 1

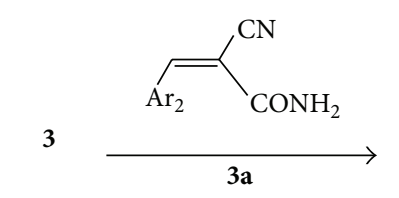

${ }_{\downarrow}^{\mathrm{O}} \mathrm{S} / \mathrm{TEA}$<smiles></smiles>

\begin{tabular}{c|cc}
$\mathbf{1 1}$ & $\mathrm{Ar}_{1}$ & $\mathrm{R}_{1}$ \\
\hline $\mathbf{a}$ & $p-\mathrm{CH}_{3} \mathrm{OPh}$ & $\mathrm{CH}_{3}$ \\
$\mathbf{b}$ & $p-\mathrm{ClPh}$ & $\mathrm{H}$
\end{tabular}

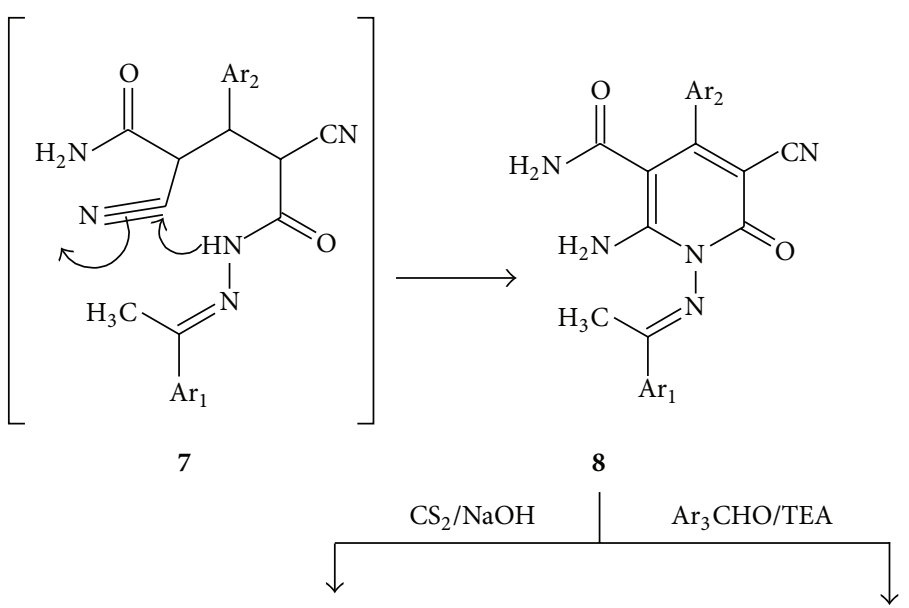<smiles>CC(C)=Nn1c(=O)c(C#N)c(C#N)c2c(=O)[nH]c(=S)[nH]c21</smiles>

10<smiles>CC(C)=Nn1c(=O)c(C#N)c(C#N)c2c(=O)[nH]c([As])nc21</smiles>

9

\begin{tabular}{ccc}
$\mathrm{Ar}_{1}$ & $\mathrm{Ar}_{2}$ & $\mathrm{Ar}_{3}$ \\
\hline$p-\mathrm{CH}_{3} \mathrm{OPh}$ & $p-\mathrm{ClPh}$ & $p-\mathrm{NO}_{2} \mathrm{Ph}$
\end{tabular}

Scheme 2 
<smiles>CCOC(=O)CSC(Nc1ccccc1)=C(C#N)C(=O)NN=C(C)c1ccc(OC)cc1</smiles>

SCHEMe 3

[18] yielding the nonisolable intermediate potassium sulfide salt 12, and then ethylchloroacetate was added affording 1,3thiazolidinone derivative 14. Probably, the reaction mechanism is assumed to proceed via S-alkylation to give the intermediate $\mathbf{1 3}$ which was cyclized to the corresponding thiazolidinone derivatives 14. Elemental analyses and spectral data were in favor of these proposed 1,3-thiazolidinone structures. The IR spectrum of $\mathbf{1 4}$ showed absorption bands at $1742 \mathrm{~cm}^{-1}$ due to thiazolidinone CO. The ${ }^{1} \mathrm{H}$ NMR spectrum showed signals in the region at $\delta 4.02 \mathrm{ppm}$ corresponding to $\mathrm{C} 4$ protons of the thiazolidinone ring. On the other hand, treatment of the nonisolable potassium sulfide salt 12 with dimethylsulfate afforded 3-anilino-2-cyano-3methylthio-acrylohydrazide 15. Cyclocondensation of the acrylohydrazide 15 with hydrazine hydrate in boiling ethanol afforded aminopyrazole derivative 16. The structure of $\mathbf{1 5}$ and $\mathbf{1 6}$ was identified as the reaction product on the basis of their elemental analysis and spectroscopic data. ${ }^{1} \mathrm{H}$ NMR spectrum of 15 displayed the following signals at $\delta 2.50$ corresponding to the $\mathrm{SCH}_{3}$ group, $\delta 10.10$ corresponding to the NHPh group, and $\delta 11.60$ corresponding to the NHCO group. IR spectrum of $\mathbf{1 6}$ showed the lacks of absorption band assignable to the $\mathrm{CN}$ group and the presence of a new absorption band at $3298,3242 \mathrm{~cm}^{-1}$ assignable to $\mathrm{NH}_{2}$ group. Its ${ }^{1} \mathrm{H}$ NMR spectrum showed signals at $\delta 6.13 \mathrm{ppm}$ corresponding to the $\mathrm{NH}_{2}$ protons, another three singlet signals at $\delta 8.00,8.20$, and $10.10 \mathrm{ppm}$ assignable to three $\mathrm{NH}$ protons (Scheme 3).

Reaction of compounds $\mathbf{3 a}, \mathbf{b}$ with carbon disulfide in boiling DMF containing catalytic amount of potassium hydroxide afforded nonisolable intermediate potassium sulfide salts $17 \mathbf{a}, \mathbf{b}$. Treatment of the nonisolable potassium salts 17a,b with dilute hydrochloric acid [19] afforded the corresponding dithiol derivatives 18a,b. The structures of compounds 18a,b were based on both elemental analyses and spectral data. The ${ }^{1} \mathrm{H}$ NMR spectrum of 18 a revealed signal at $\delta 2.73$ and $2.88 \mathrm{ppm}$ for two $\mathrm{SH}$ protons and at $\delta$ $10.50 \mathrm{ppm}$ for NHCO proton. Moreover, Alkylation of dithiol derivatives $\mathbf{1 7 a}, \mathbf{b}$ with ethylchloroacetate yielded the corresponding ethyl [2-cyano-1-mercapto-3-oxoprop-1-enyl) thio] acetate derivatives 19a,b [20]. The structures of $\mathbf{1 9 a}, \mathbf{b}$ were supported on the basis of elemental analyses and spectral data. Ketene S,S-dithio-acetals derivatives 20a,b can be prepared by alkylation of dithiol derivatives $17 \mathbf{a}, \mathbf{b}$ with dimethylsulfate. The structure of $\mathbf{2 0}$ was elucidated on the basis of the elemental analyses and spectral data. The IR spectrums of 20a showed the appearance of absorption band at 3203 for $\mathrm{NH}$ and $2256 \mathrm{~cm}^{-1}$ for $\mathrm{CN}$ groups. ${ }^{1} \mathrm{H} \mathrm{NMR}$ spectrum of 20b showed singlet signal at $\delta 2.50 \mathrm{ppm}$ for 6 protons of two similar methyl protons, while a singlet signal for the methylene protons disappeared.

Cyclocondensation of bis-(methylthio)acrylohydrazide derivative 20a with hydrazine derivatives such as hydrazine hydrate and 2-hydrazino-1,3-benzothiazole affords the corresponding 5-(methylthio) 1H-pyrazole-4-carbohydrazide 21 and 22, respectively. The structures of compounds $\mathbf{2 1}$ and $\mathbf{2 2}$ were as established and confirmed as the reaction product on the basis of their elemental analyses and spectral data. The IR spectrum of 21 showed absorption band at $3300,3243 \mathrm{~cm}^{-1}$ assignable for $\mathrm{NH}_{2}$, in addition to disappearance of nitrile function signal. Its ${ }^{1} \mathrm{H}$ NMR spectrum revealed the presence of singlet signals at $\delta 6.13 \mathrm{ppm}$ assignable to the $\mathrm{NH}_{2}$ protons (Scheme 4).

3.2. Antitumor Activity. Some newly synthesized compounds 3a, 3b, 4a, 4b, 6a, 6b, 11b, 14, 15, 16, 18b, 19a, 19b, 21, and 22, screened in vitro against human breast cancer cell line (MCF7), using doxorubicin (Doxo) as a reference drug. The results 


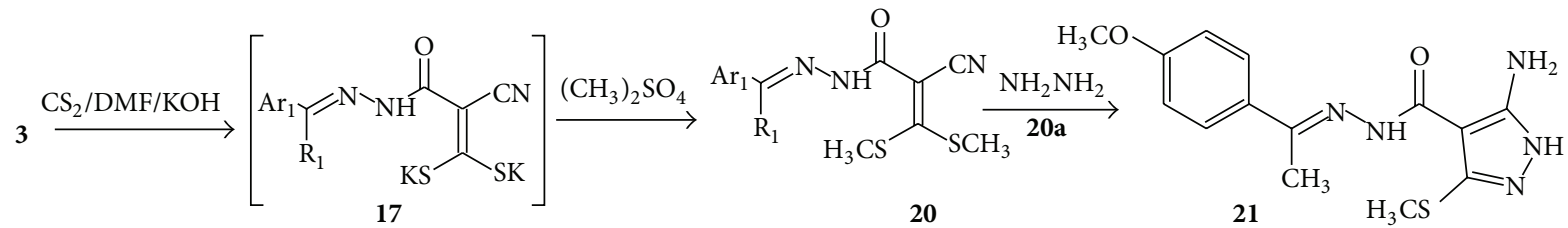<smiles>[R1]C([Y18])=NNC(=O)/C(C#N)=C(/S)SCC(=O)OCC</smiles>

19

\begin{tabular}{c|cc} 
17-20 & $\mathrm{Ar}_{1}$ & $\mathrm{R}_{1}$ \\
\hline $\mathbf{a}$ & $p-\mathrm{CH}_{3} \mathrm{OPh}$ & $\mathrm{CH}_{3}$ \\
$\mathbf{b}$ & $p-\mathrm{ClPh}$ & $\mathrm{H}$
\end{tabular}<smiles>NNc1nc2ccccc2s1</smiles><smiles>[R1]C([Y18])=NNC(=O)C(C#N)=C(S)S</smiles>

18<smiles>COc1ccc(/C(C)=N/NC(=O)c2c(S)nn(-c3nc4ccccc4s3)c2N)cc1</smiles>

22

SCHeme 4

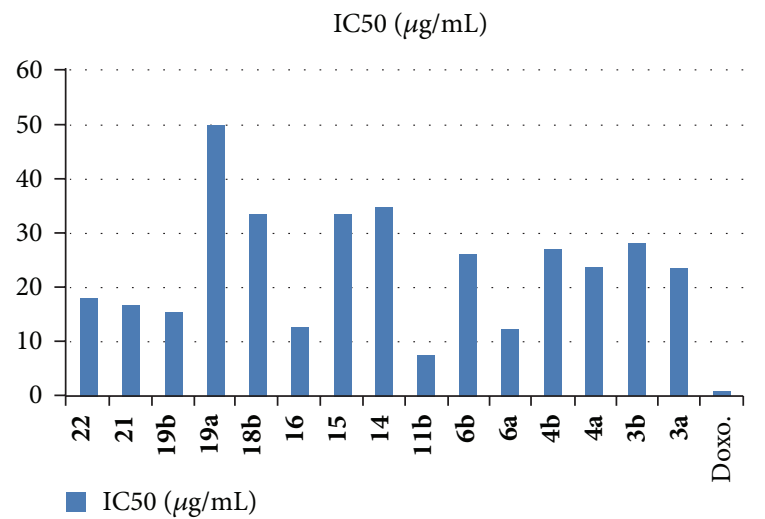

FIGURE 1: In vitro anticancer screening of the synthesized compounds against human breast cancer cell line (MCF-7).

were compared to the antiproliferative effects of the reference control doxorubicin (Table 1, plotted in Figure 1). The results indicated that most compounds demonstrated substantial growth inhibitory effects against the human tumor cells at the tested concentrations. The antiproliferative activity of the test compounds against tumor cell lines may be arranged in a descending order due to measured concentration required to inhibit tumor cell proliferation by IC $50 \mu \mathrm{g} / \mathrm{mL}$ which corresponds to the concentration required for $50 \%$ inhibition of cell viability. In general, compounds $11 b, 6 a, 16,19 b, 21$, and 22 with IC50 values $7.5,12.2,12.6,15.3,16.1$, and $18 \mu \mathrm{g} / \mathrm{mL}$, respectively, showed significant activity on the tumor cell lines tested.

\section{Conclusions}

In this work, cyanoacetylhydrazine (1) reacted with 4-methoxy-acetophenone and 4-chlorobenzaldehyde $(\mathbf{2 a}, \mathbf{b})$ to afford the hydrazide-hydrazone derivatives, $\mathbf{3 a}, \mathbf{b}$. The latter was reacted with different reagents to give coumarin, pyridine, thiophene, and pyrazole derivatives. The antitumor evaluations of the newly synthesized products were carried out, showing that compound exhibited moderate activity.

\section{References}

[1] J. V. Ragavendran, D. Sriram, S. K. Patel et al., "Design and synthesis of anticonvulsants from a combined phthalimide-GABAanilide and hydrazone pharmacophore," European Journal of Medicinal Chemistry, vol. 42, no. 2, pp. 146-151, 2007.

[2] A. G. M. Fraga, C. R. Rodrigues, A. L. P. de Miranda, E. J. Barreiro, and C. A. M. Fraga, "Synthesis and pharmacological evaluation of novel heterotricyclic acylhydrazone derivatives, designed as PAF antagonists," European Journal of Pharmaceutical Sciences, vol. 11, no. 4, pp. 285-290, 2000.

[3] A. R. Todeschini, A. L. P. de Miranda, K. C. M. da Silva, S. C. Parrini, and E. J. Barreiro, "Synthesis and evaluation of analgesic, antiinflammatory and antiplatelet properties of new 2pyridylarylhydrazone derivatives," European Journal of Medicinal Chemistry, vol. 33, no. 3, pp. 189-199, 1998.

[4] G. A. Silva, L. M. M. Costa, F. C. F. Brito, A. L. P. Miranda, E. J. Barreiro, and C. A. M. Fraga, "New class of potent antinociceptive and antiplatelet $10 \mathrm{H}$-phenothiazine-1-acylhydrazone derivatives," Bioorganic and Medicinal Chemistry, vol. 12, no. 12, pp. 3149-3158, 2004.

[5] S. Gemma, G. Kukreja, C. Fattorusso et al., "Synthesis of N1arylidene-N2-quinolyl- and N2-acrydinylhydrazones as potent antimalarial agents active against CQ-resistant $P$. falciparum strains," Bioorganic and Medicinal Chemistry Letters, vol. 16, no. 20, pp. 5384-5388, 2006.

[6] G. Turan-Zitouni, Y. Blache, and K. Güven, "Synthesis and antimicrobial activity of some imidazo-[1,2-a]pyridine-2-carboxylic acid arylidenehydrazide derivatives," Bollettino Chimico Farmaceutico, vol. 140, no. 6, pp. 397-400, 2001.

[7] E. Gürsoy and N. Güzeldemirci-Ulusoy, "Synthesis and primary cytotoxicity evaluation of new imidazo[2,1-b]thiazole derivatives," European Journal of Medicinal Chemistry, vol. 42, no. 3, pp. 320-326, 2007. 
[8] S. A. M. El-Hawash, A. E. Abdel Wahab, and M. A. El-Demellawy, "Cyanoacetic acid hydrazones of 3-(and 4)acetylpyridine and some derived ring systems as potential antitumor and antiHCV agents," Archiv der Pharmazie, vol. 339, no. 1, pp. 14-23, 2006.

[9] A. G. Silva, G. Zapata-Sudo, A. E. Kummerle, C. A. M. Fraga, E. J. Barreiro, and R. T. Sudo, "Synthesis and vasodilatory activity of new $N$-acylhydrazone derivatives, designed as LASSBio-294 analogues," Bioorganic and Medicinal Chemistry, vol. 13, no. 10, pp. 3431-3437, 2005.

[10] M. T. Abdel-Aal, W. A. El-Sayed, and E. H. El-Ashry, "Synthesis and antiviral evaluation of some sugar arylglycinoylhydrazones and their oxadiazoline derivatives," Archiv der Pharmazie, vol. 339, no. 12, pp. 656-663, 2006.

[11] A. Monks, D. Scudiero, P. Skehan et al., "Feasibility of a highflux anticancer drug screen using a diverse panel of cultured human tumor cell lines," Journal of the National Cancer Institute, vol. 83, no. 11, pp. 757-766, 1991.

[12] A. M. Khalil, M. A. Berghot, and M. A. Gouda, "Synthesis and antibacterial activity of some new heterocycles incorporating phthalazine," European Journal of Medicinal Chemistry, vol. 44, no. 11, pp. 4448-4454, 2009.

[13] T. M. Abu Elmaati and F. M. A. El-Taweel, "Routes to pyrazolo[3,4-e][1,4] thiazepine, pyrazolo[1,5-a]pyrimidine and pyrazole derivatives," Journal of the Chinese Chemical Society, vol. 50, no. 3, pp. 413-418, 2003.

[14] H. Z. Shams, R. M. Mohareb, M. H. Helal, and A. E. Mahmoud, "Novel synthesis and antitumor evaluation of polyfunctionally substituted heterocyclic compounds derived from 2-cyano- $N$ (3-cyano-4,5,6,7-tetrahydrobenzo[b]thiophen-2-yl)-acetamide," Molecules, vol. 16, no. 1, pp. 52-73, 2011.

[15] M. S. Al-Said, M. S. Bashandy, S. I. Al-Qasoumi, and M. M. Ghorab, "Anti-breast cancer activity of some novel 1,2-dihydropyridine, thiophene and thiazole derivatives," European Journal of Medicinal Chemistry, vol. 46, no. 1, pp. 137-141, 2011.

[16] A. A. Bekhit, H. T. Y. Fahmy, S. A. F. Rostom, and A. M. Baraka, "Design and synthesis of some substituted $1 \mathrm{H}$-pyrazolyl-thiazolo[4,5- $d]$ pyrimidines as anti-inflammatory-antimicrobial agents," European Journal of Medicinal Chemistry, vol. 38, no. 1, pp. 27-36, 2003.

[17] R. M. Scrowston, "Recent advances in the chemistry of benzo [b] thiophenes," Advances in Heterocyclic Chemistry, vol. 29, pp. 171-249, 1981.

[18] M. A. Gouda and A. A. Abu-Hashem, "Synthesis, characterization, antioxidant and antitumor evaluation of some new thiazolidine and thiazolidinone derivatives," Archiv der Pharmazie, vol. 344, no. 3, pp. 170-177, 2011.

[19] A. A. Faddal, M. M. Mukhtar, and H. M. Refat, "Utility of activated nitriles in the synthesis of some new heterocyclic compounds," American Journal of Organic Chemistry, vol. 2, no. 2, pp. 32-40, 2012.

[20] G. H. Elgemeie, A. H. Elghandour, A. M. Elzanaty, and S. A. Ahmed, "Novel 1,3-dithiolan-2-ones using sodium $\alpha$-cyanoketene dithiolates," Synthetic Communications, vol. 36, no. 6, pp. 755-764, 2006. 

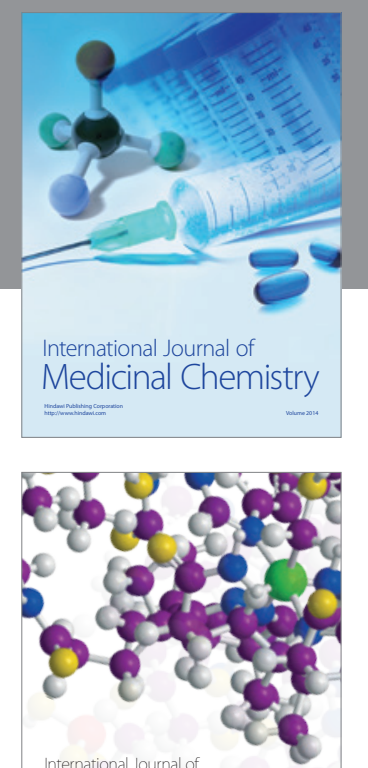

\section{Carbohydrate} Chemistry

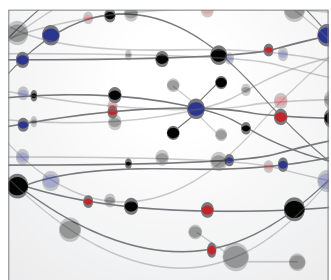

The Scientific World Journal
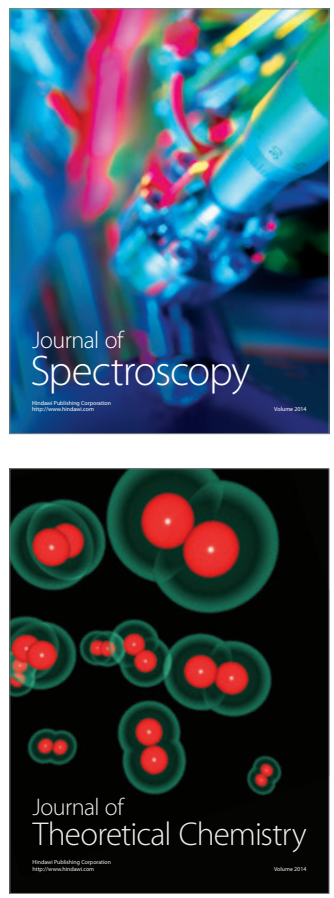
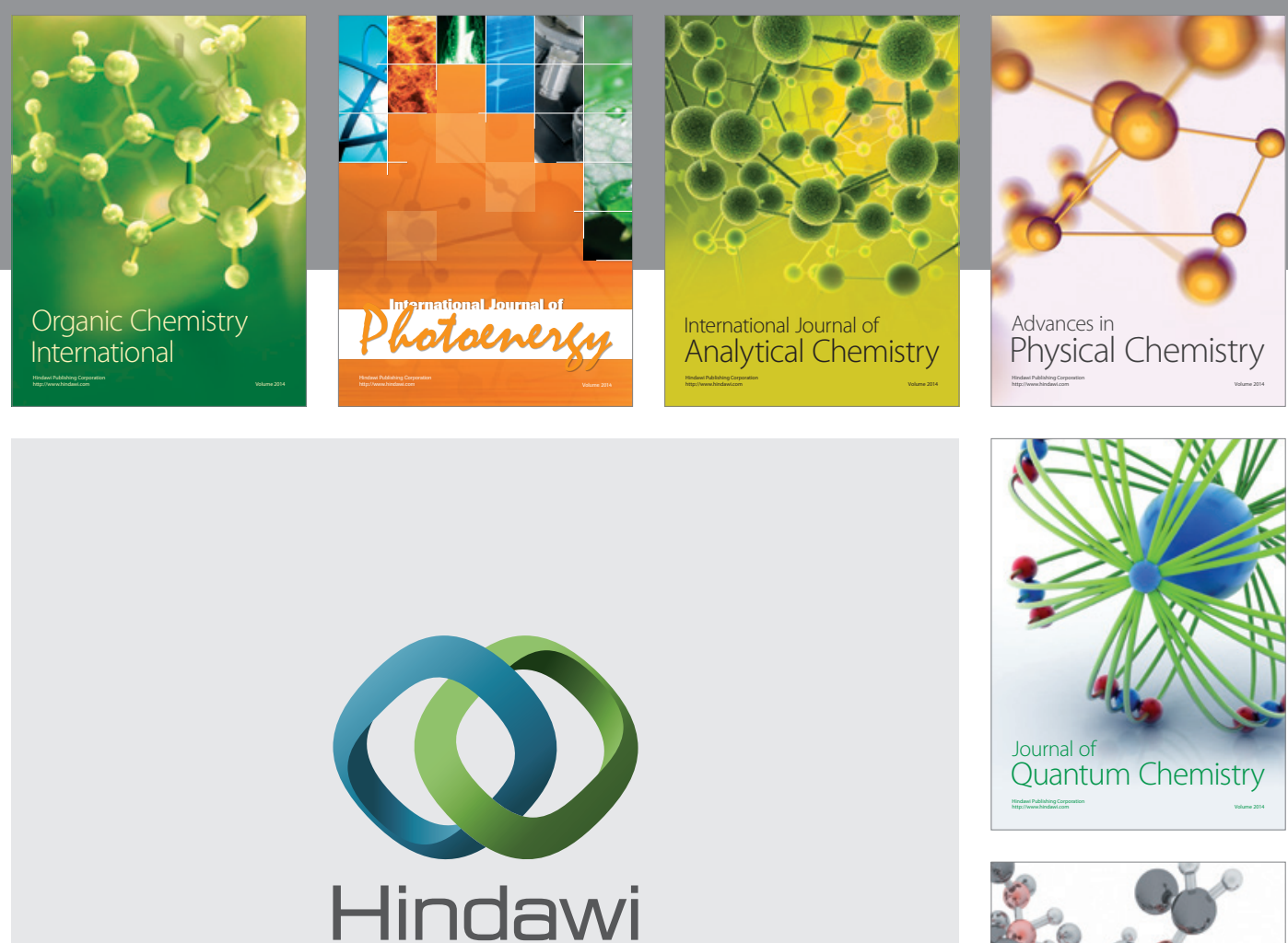

Submit your manuscripts at

http://www.hindawi.com

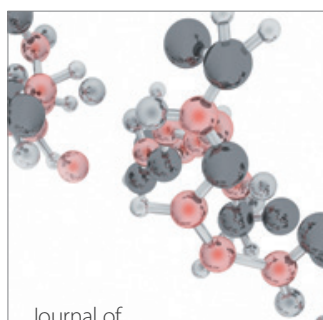

Analytical Methods

in Chemistry

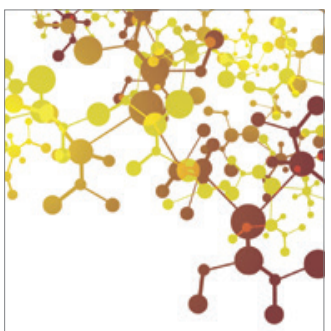

Journal of

Applied Chemistry

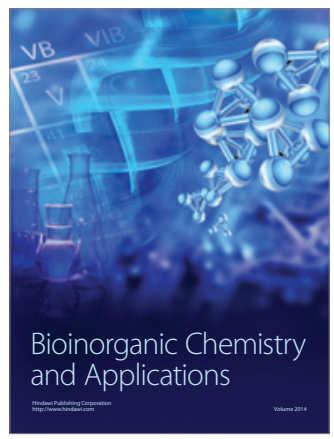

Inorganic Chemistry
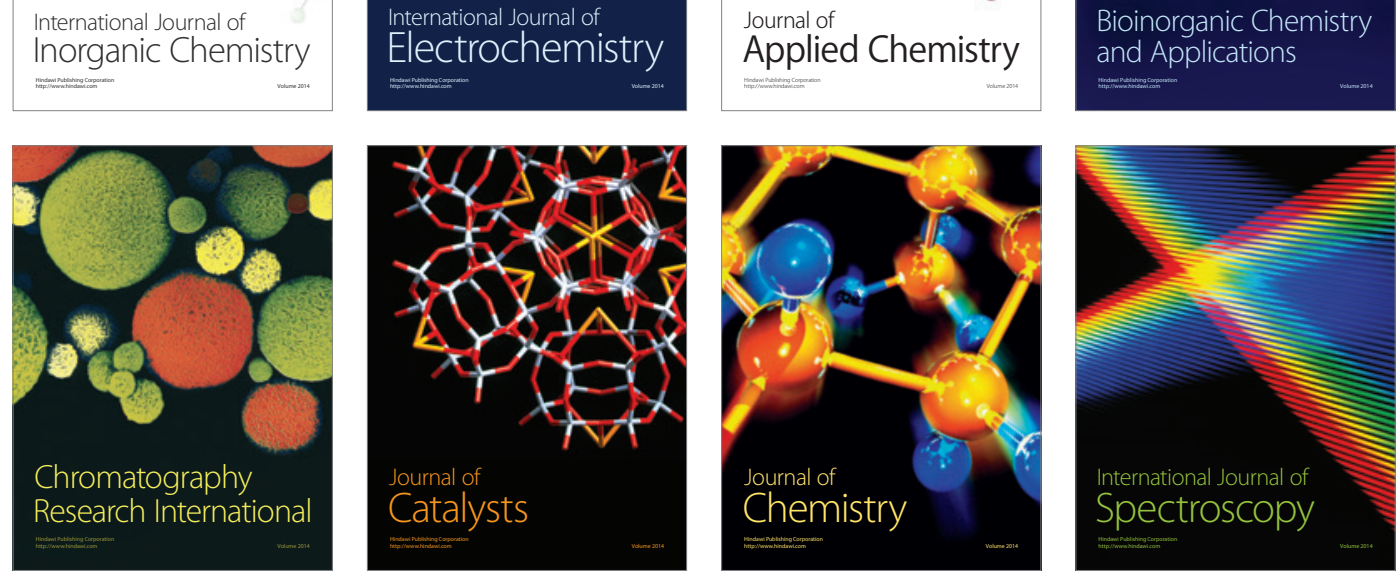